diagnosis of AGE. Stool samples were obtained for rotavirus testing and genotype investigation using ELISA and multiplex RT-PCR.

Results The prevalence of rotavirus infection was $27.3 \%$ (95\% CI $23.6-31)$. Infants $<2$ years of age were most frequently affected $(91.6 \%)$. The most dominant rotavirus genotype was G3P [8], which accounted for $40.4 \%$ of cases. On multivariate analysis, rotavirus was significantly associated with the episode occurring in the winter season (aOR 6.73; 95\% CI 3.45-3.31), vomiting (aOR 3.05; 95\% CI 1.37-6.75), fever (aOR 1.84; 95\% CI 1.10-3.07) and dehydration (aOR 8.20; 95\% CI 3.45-19.47).

Conclusion The determination of rotavirus infection prevalence and its risk factors will help us to better understand the epidemiology of the disease in our country in order to develop effective preventive measures, including vaccines.

\section{EVALUATION OF MEASLES OUTBREAK DURING 2010/2011 IN SKOPJE, MACEDONIA}

doi:10.1136/archdischild-2012-302724.0935

'Z Arsenievski, 'L Lazarevska, 'B Danilovska, 'L Badeva, 'L Sinadinovska, 'A Stojanov, 'M Manev, ${ }^{2}$ E Zisovska. 'Epidemiology, Center for Public Health; ${ }^{2}$ Neonatology, Obstetrics and Gynaecology Clinic Skopje, Skopje, FYR Macedonia

Background Due to low rates of vaccination coverage, in mostly rural in Skopje and as a result of military conflict in 2001, lead to spillover of the measles from neighboring countries, where outbreak of measles was already declared.

Methods and materials: Measles reporting is mandatory in Macedonia. Cases analyzed had to meet the national case definition. Case-series investigation were conducted, surveys of rates of vaccination coverage.

Results From 07.09.2010 to 22.07.2011, we have registered 596 cases of measles. Of these 596, twenty five case after getting a negative result from laboratory testing were discarded, so the number of cases of measles in the area of Skopje was $572(\mathrm{Mb}=97.0 / 100.000)$. The first case was during a 13 months of age unvaccinated child. Out of 572 cases of measles 235 (41.0\%) were hospitalized, mostly with severe clinical symptoms. According to the patients vaccination status the conclusion was that: 517 (90.4\%) persons were vaccinated, of which 59 not subject to the vaccination, $19(3.3 \%)$ persons no data, $36(6.3 \%)$ persons were vaccinated, of which a portion of MMR are 30 and 6 with two doses. During the outbreak, laboratory confirmed 84 cases out of 103 taken materials, were positive.

Conclusions The high rate of vaccine coverage in most municipalities in Skopje, unvaccinated children with a first dose and absence of the second dose in the first grade in elementary school, mostly in rural areas affected by the military conflict in 2001, were the cause of measles in epidemic form.

\section{PREVALENCE AND MANIFESTATIONS OF INFECTIOUS MONONUCLEOSIS-LIKE SYNDROME IN A CHILDREN'S HOSPITAL IN GREECE}

doi:10.1136/archdischild-2012-302724.0936

${ }^{1} \mathrm{C}$ Koutsaftiki, ${ }^{2} \mathrm{M}$ Mammas, ${ }^{2} \mathrm{SA}$ Kassina, ${ }^{2} \mathrm{~A}$ Kapetanaki, ${ }^{2} \mathrm{E}$ Kotsari, ${ }^{3} \mathrm{~A}$ Makri, ${ }^{3} \mathrm{~A}$ Voyatzi, ${ }^{2 M}$ Kousouri. 'Pediatrics, Penteli's General Children's Hospital; ${ }^{2} 1$ st Pediatric Department, Penteli's Children's Hospital; ${ }^{3}$ Department of Clinical Microbiology and Immunology, Penteli's Children's Hospital, Athens, Greece

Introduction Infectious Mononucleosis Syndrome (IMS), is characterized by fever, lymphadenopathy, tonsillitis, hepatomegaly, spleenomegaly. Also peripheral lymphocytosis with $>10 \%$ atypical lymphocytes is present. It is attributed mostly to Epstein-Barr virus (EBV), less to Cytomegalovirus (CMV) and rarely to Herpes Simplex Virus (HSV) and others.
Occasionally, atypical manifestations and serious complications might occur.

Objective Estimating the prevalence of the disease, the responsible infectious agents and of the typical and atypical manifestations in pediatric populations.

Material and Methods Within 12months, 700 serum samples were examined, from children 1-15 years old. Specimens' laboratory investigation included: 1) indirect immunofluorescence, detecting IgG-IgM antibodies against EBV's Viral Capsid Antigen (VCA), 2) Immuno Chemistry luminescence determination of CMV's IgGIgM antibodies, 3) EIA, identifying HSV's IgG-IgM antibodies.

Results Typical clinical findings were: prolonged fever (89\%), lymphadenopathy (82\%), tonsillitis $(57 \%)$, hepato-spleenomegaly $(40 \%)$, cough $(20 \%)$ and skin eruption $(10 \%)$. Atypical manifestations were: menimgoencephalitis in 2 children, 22 months and 7 years old (caused by EBV and HSV1 respectively), haemophagocytic lymphoistiocytosis in an 8 year old (by EBV), Gianotti-Crosti syndrome in an 18 months old (by EBV) and pneumonia in a 22 months old (by EBV). In meningoencephalitis, viral DNA was detected using PCR in cerebrospinal fluid while in the remaining cases, positive IgM antibodies were found. Of the 700 children examined, 56 (8\%) had positive serology for EBV, 29 (4.14\%) for CMV and 1 for HSV 1.

Conclusions IMS is often amongst Greek children. $65 \%$ of the cases are attributed to EBV and 35\% to CMV which is in accordance to the international literature.

\section{STUDY OF DETECTION VIBRIO CHOLERAE 01 FROM KAROON RIVER WATERS AHVAZ AND ROLE IN THE PUBLIC HEALTH}

doi:10.1136/archdischild-2012-302724.0937

M Mehdinejad, N NassirAbadi, H Godarzi. Ahvaz Jundishapur University of Medical Sciences (AJUMS), School of Medicine, Dept. of Microbiology, Ahvaz, Iran

Background The watershed of Dez and Karoon rivers located in middle Zagrous mountain with area about 68481-, Thus watershed is a part of Persian gulf watershed. Cholera, an acute intestinal infection caused by the bacterium Vibrio cholerae ( $V$. cholerae) is a historically feared epidemic diarrheal disease that remains a major public health problem in many parts of Africa, Asia, and Latin America. $V$. cholerae $\mathrm{O} 1$ exists as two major serotypes, Inaba and Ogawa, a member of the family Vibrionaceae is transmitted through Fresh water contaminated with fecal matter. Foodborne infections have been traced to raw. The target of study the Segregate $V$. cholerae 01 (Vibrionaceae) in the Karoon Ahvaz River.

Methods In four stages (April, to July 2010), a total 100 samples of water from Karoon River Ahvaz were collected. During the study period the recorded river temperature was about $25-28^{\circ} \mathrm{C}$ and $\mathrm{pH}$ ranged from 7 to 8 . Swabs were cultured onto thiosulphate citrate bile sucrose and MacConkey, and morphological colonies compatible with Vibrio were characterized by oxidase test and agglutinated with antiserum for serotype determination. Also $V$. cholerae biochemical tests with API 20E.

Results From 100 samples of water Karoon River in Ahvaz, Iran, 8 $(8 \%)$ sample were positive for Vibrio cholerae strains. The isolated strains from water Karoon River in Ahvaz, Iran, were Vibrio cholerae O1 (inaba).

Conclusion The priorities for cholera control remain public health interventions through improved water and sanitation, improved surveillance and access to health care facilities, and further development of appropriate vaccines. 
D Nikčević, D Terzić, B Dupanović, B Andrić. Clinic for Infectious Diseases, Clinical Center of Montenegro, Podgorica, Montenegro

Background and Aims One third of the world population has been infected by the hepatitis B virus (HBV), causing an enormous worldwide burden of chronic hepatitis, cirrhosis and hepatocellular carcinoma. We make revue of clinical characteristics of acute hepatitis B infection in children in Montenegro.

Methods 180 children suffering from acute virus hepatitis were examined on Clinic for infectious diseases in Podgorica during the period 2006-2012. We used epidemiological and clinical data and blood tests for hepatitis viruses, Epstein-Barr virus, CMV and adenoviruses. Research sample is consisted of 18 children, which are old from 7-15 years with clinical, laboratory and virology verification of acute hepatitis $B$.

Results Epidemiological data have indicated the infection trough blood or blood products in 14 children and in 4 the way of infection remained unknown. HBsAg and antiHBc IgM were positive, values of alanine aminotransferase were elevated and PCR HBV DNK was higher than $200000 \mathrm{U} / 1$ in all children. The most frequent symptoms were the loss of appetite (88\%) and fatigue (70\%). Fever was registered in $30 \%$ of children, while the diarrhea is reported in $25 \%$, hepatomegaly in $86 \%$, enlarged spleen in $20 \%$ and icterus in $60 \%$ of cases. Patients without symptoms were present in $12 \%$ of cases. Three children after a follow-up longer than one year developed chronic infection.

Conclusions Even though immunisation for hepatitis B virus is mandatory since 2002 in Montenegro, the acute hepatitis B isn't rare infection. Children are at the greatest risk for developing potentially fatal complications of the infection.

\section{EPIDEMILOGIC STUDY ON PATHOGENIC BACTERIA CAUSING DIARRHEA IN CHILDREN AND DETECTION OF ANTIBIOTICS RESISTANCE PATTERNS IN HAMADAN, WEST OF IRAN}

doi:10.1136/archdischild-2012-302724.0939

'R Yousefimashouf, ${ }^{2} \mathrm{~F}$ Eghbalian, ${ }^{3} \mathrm{M}$ Koshki. 'Department of Microbiology; ${ }^{2}$ Dept. of Pediatrics; ${ }^{3}$ Hamadan University of Medical Sciences, Hamadan, Iran

Background and Aim Bacterial diarrhea is very common in particular in devoloping countries and is still one of the most causes of mortalities in children. The aim of present study was to identify the most common of bacterial agents causing acute bacterial diarrhea in children under 14 years old and detection of their resistance to antibiotics.

Methods During two years, 610 samples obtained from children under 14 years old with gastroentrotitis were investigated for bacterial cultures, frequency of age, serogrouping of isolates and antibiogram patterns. Polyvalent (I, II, III, IV) and monovalant antisera were used for serogrouping of E. coli (EPEC). Antibiogram tests were also performed by gel-diffusion method of Kirby-Bauer. The data were gathered through a questionnaire and analysed using spss software.

Results Out of 610 tested samples, 155 cases (25.4\%) had positve culture for intestinal pathogenic bacilli. The most common isolate was; Escherichia coli (EPEC) with 105 cases (67.8\%) and the lowest isolate was Shigella with 18 cases $(11.6 \%)$. The most common serogroup of E. coli was $0128(26.6 \%)$ and the lowest serogroup was 0119 $(5.7 \%)$. The most common serogroups of Salmonella were S. typhi $(34.4 \%)$ and $S$. typhimurium. The most common serogroup of Shigella was $S$. sonnei $(55.6 \%)$. The most effective antibiotics against bacteria were ceftriaoxne, nitrofurantoin, imipenem, amikacin and gentamycin.

Conclusions The present study showed that Escherichia coli (EPEC) and Salmonella species are predominant causes of bacterial diarrhea in children under 14 years old in this region. Most species showed high resisitance to routine antibiotics such as ampicillin, trimethoprim and chloramphenicol.

\section{CLINICAL SPECTRUM OF RESPIRATORY TRACT INFECTIONS: A STUDY AMONG CHILDREN IN AJMAN, UAE}

doi:10.1136/archdischild-2012-302724.0940

${ }^{1} \mathrm{M}$ Hassan, ${ }^{2} \mathrm{LJ}$ John, ${ }^{3} \mathrm{M}$ Hassan. 'Pharmaceutics; 2 Pharmacology, Gulf Medical University; ${ }^{3}$ Pediatrics, Gulf Medical College Hospital, Ajman, United Arab Emirates

Background and Aims Respiratory tract infection (RTI) among children is one of the most common reasons for physician visits and hospitalization and associated with significant morbidity. It is a leading cause of sickness among school children and parental absenteeism from work. Accordingly, this study was conducted to evaluate the spectrum of the respiratory tract infections among the children attending the outpatient department (OPD) of pediatrics in GMC Hospital, Ajman.

Methods A cross-sectional study was conducted from the medical records of pediatric patients (aged 0-12 years) diagnosed with RTIs during January 2011 at GMC Hospital, Ajman. The demographic data and the clinical diagnosis were analyzed (descriptive statistics) using SPSS version 19.

Results A total of 2416 patients attended the OPD of pediatrics during the study period of which RTI accounted for $20.2 \%$ ( $n=488)$. $55.5 \%(n=271)$ were males and $44.5(n=217)$ were females. Majority of the patients were Egyptians followed by Emiratis and Indians. Forty six percent of the patients (225) were between 1-5 years of age. The four most commonly diagnosed RTIs were unspecified upper respiratory tract infections (URTI) (20.3\%), common cold combined with bronchitis (17.8\%), tonsillitis (8.8\%), followed by bronchitis (8\%) in rank order. URTI was most commonly presented by children of $1-5$ years $(50.5 \%)$, while common cold combined with bronchitis was frequent among those below 1 year (47.1\%).

Conclusions Comparable to previous reports upper respiratory tract infections were the most common RTI among this population and children of 1-5 years were most vulnerable to RTI

\section{AUTONOMIC DYSFUNCTION IN INFANTS WITH RSV INFECTION}

doi:10.1136/archdischild-2012-302724.0941

${ }^{1} \mathrm{C}$ Liebrand, 'M Bouwman, ${ }^{2} \mathrm{I}$ Ahout, ${ }^{3} \mathrm{M}$ Kox, ${ }^{1} \mathrm{C}$ Neeleman. ${ }^{1}$ Pediatric ICU; ${ }^{2}$ Department of infectious diseases, ${ }^{3} I C U$ research department, UMCN St Radboud, Nijmegen, The Netherlands

Background Apnea can be a presenting symptom in respiratory Synctial Virus (RSV) infection. The incidence of apnea in RSV infected infants varies between 16 and $25 \%$ with a particularly high risk associated with young age $(<3 \mathrm{mo})$ and prematurity. Apnea is frequently observed in early RSV infection when symptoms are still confined to the upper airways, suggesting inflammatory response induced autonomic dysfunction.

Objective The aim of the study is to assess autonomic dysfunction as indexed by Heart Rate Variability (HRV) in severe RSV infection. The secondary parameter is to analyze the effect of other viral pathogens on autonomic function in infants with LRTI requiring mechanical ventilation.

Methods Prospective observational study in a tertiary PICU in infants with viral LRTI requiring mechanical ventilation. Controls were age matched infants without signs of infection. Autonomic function was assessed via HRV, monitoring low frequency (LF) and high frequency (HF) indices of short time recordings (5 min).

Results In 7 patients and 13 controls 36 recording were analyzed. Total HRV, LF and LF/HF ratios were significant lower in RSV patients versus controls $(p<0.0045, p<0.0002$ and $p<0.0001$ respectively). In ventilated infants with LRTI caused by other respiratory 